\title{
Inspecting aviation composites at the stage of airplane manufacturing by applying 'classical' active thermal NDT, ultrasonic thermography and laser vibrometry
}

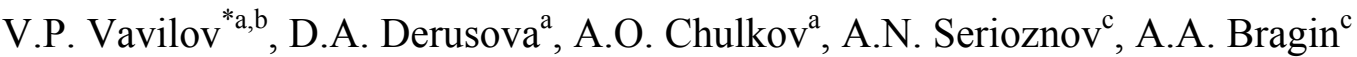 \\ aTomsk Polytechnic University, 7 Savinykh St., Tomsk, Russia 634028; \\ ${ }^{\mathrm{b}}$ Tomsk State University, 36 Lenin Av., Tomsk, Russia 634050; \\ 'Siberian Aeronautical Research Institute named after S.A. Chaplygin, \\ 21 Polzunov St., Novosibirsk, Russia 630051
}

\begin{abstract}
The results of applying three nondestructive testing techniques to the inspection of parts of a new Russian TVS-2DTS airplane made of carbon fiber reinforced plastic are presented. A basic technique implemented in workshop conditions implements optical stimulation of inspected parts. The usefulness of ultrasonic infrared thermography combined with laser vibrometry in the evaluation of parts with complicated geometry is illustrated. Samples with artificial and real defects have been tested in workshop conditions.
\end{abstract}

Keywords: infrared thermography, composite, carbon fiber reinforced plastic, ultrasonic stimulation, laser vibrometry

\section{INTRODUCTION}

Composites [1, 2] are being increasingly used in the Russian aviation industry. In 2017, a new TVS-2DTS regional airplane fully made of graphite epoxy composite (GEC) components was demonstrated alive at the MAKS'2017 aviation show in Moscow. A manufacturer of this airplane - Siberian Aeronautical Research Institute named after S.A. Chaplygin - uses ultrasonics as a primary nondestructive testing (NDT) technique in the workshop environment, while active thermal NDT has been accepted as a complementary inspection method. Tomsk Polytechnic University is involved into development of a modular thermal NDT device which will allow implementation of both one- and two-side test procedures on components of various geometries. A basic technique involves standard optical heating with halogen lamps which are mechanically turned aside after heating stopped in order to avoid residual thermal radiation reflected from the surface of inspected samples. The second thermal NDT technique is based on using a high-power magnetostrictive ultrasonic device (frequency $22 \mathrm{kHz}$, electrical power up to $1.5 \mathrm{~kW}$ ) to stimulate 'kissing' defects in components under test. Both techniques should be implemented in the workshop conditions. Finally, laser vibrometry combined with low-power resonant ultrasonic stimulation is considered as a laboratory inspection method of which practical feasibility is yet to be explored. However, this technique has already proven to be efficient and illustrative in detecting delaminations in ribs made of graphite epoxy composite. Comparative results of applying three abovementioned techniques to the inspection of TVS-2DTS airplane parts will be presented in this paper.

\section{EXPERIMENTAL SETUPS}

A basic experimental setup (Figure 1a) included a special optical heating device consisted of 2 halogen lamps with the power of $1 \mathrm{~kW}$ each. An operation protocol of this device envisaged, first, the turning of the lamps aside for 3-10 seconds (adjustable) in order to let the lamps reach a work regime, i.e. provide a maximal optical power. Then the lamps

*vavilov@tpu.ru; phone 79138219749

Thermosense: Thermal Infrared Applications XL, edited by Douglas Burleigh,

Proc. of SPIE Vol. 10661, 106610W · C $2018 \mathrm{SPIE} \cdot \mathrm{CCC}$ code:

$0277-786 \mathrm{X} / 18 / \$ 18 \cdot$ doi: $10.1117 / 12.2304706$

Proc. of SPIE Vol. 10661 106610W-1 
are turned back to illuminate surface of test samples for other 3-20 s. As the lamps are switched off, they turn aside again to prevent reflected noisy radiation. Such protocol is particularly appropriate in the case of light-color and/or shiny surfaces which easily reflect incident radiation. Infrared (IR) image sequences are recorded in a standard way by means of a FLIR 325 (or Optris PI 340) IR module. All test parameters, such as a heating time and acquisition interval, as well as the number of recorded images, are controlled by a home-made computer program. This driving program is compatible with a main program which incorporates both modeling and data processing software.

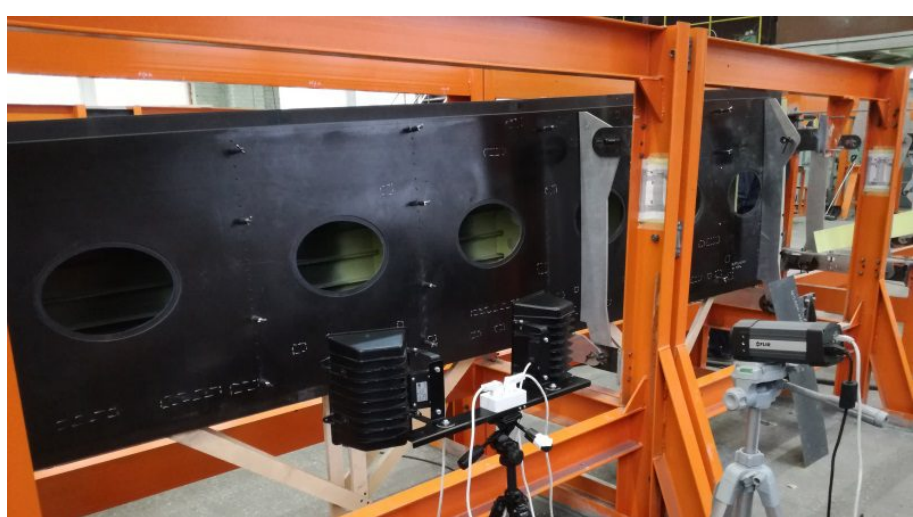

a)

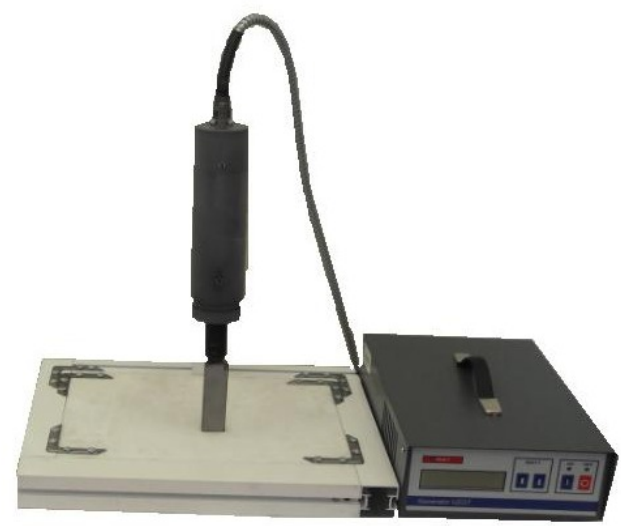

b)

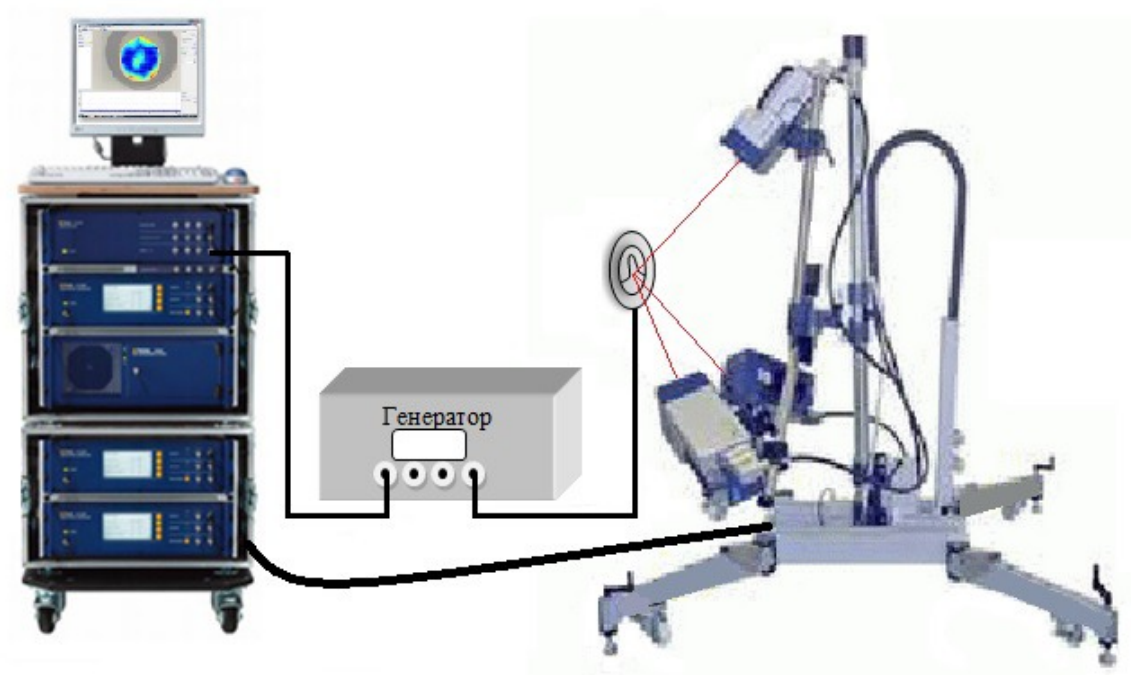

c)

Figure 1. Experimental setups used in NDT of composite-made aviation components: a - basic setup (inspecting GEC wing part, sweeping halogen lamps), b - magnetostrictive device used in IR ultrasonic thermography, c - laser vibrometry system (Polytec PSV-400-3D laser vibrometer).

Unlike many similar investigations, a setup used for performing IR ultrasonic thermography involves the use of a magnetostrictive device as a source of powerful ultrasonic waves (Figure 1b). The magnetostrictive indentor represents a $0.5 \mathrm{~mm}$-diameter steel sphere of which displacement is $\pm 7 \mu \mathrm{m}$ with the frequency of $22 \mathrm{kHz}$. The maximum electric power that is released in the coil reached $600 \mathrm{~W}$, and another version of this device may supply up to $2.5 \mathrm{~kW}$ of electric power. The estimating of the ultrasonic power injected into a sample is still questionable although it is worth stating that defect-free samples made of composites and placed on a free support have revealed no detectable mean temperature rise during ultrasonic stimulation thus proving that injected ultrasonic waves are dispersed in the environment and perform no work of heat generation. However, injecting ultrasonic waves of such high power may cause damage of a test material 
at a point of contact. To avoid this undesirable phenomenon, we used $10 \mathrm{~mm}$-diameter steel disks placed between the material and the indentor. A typical duration of ultrasonic stimulation has been from 5 to 15 seconds, and the location of a point of stimulation has been optimized in each particular test case. It is important noting that IR ultrasonic inspection by using a high-power magnetostrictive device can be hardly implemented in a workshop because of difficulties related to providing a necessary contact between the indentor and test samples, particularly, if a sample is of a complicated geometry. The corresponding work is on the agenda.

The idea of using laser vibrometry in this research appeared after we have begun studying a technique of Local Defect Resonance (LDR) first proposed by Solodov et al. [3]. This technique is characterized by a lower excitation power (a few Watts) in regard to stimulation with a single-frequency magnetostrictive transducer for which electric power may reach some $\mathrm{kW}$. Piezoelectric transducers were glued to the sample surface with epoxy adhesive to ensure efficient injection of acoustic energy into materials. The sample amplitude-frequency characteristics of vibrations were obtained at each sample point in the frequency range from 0 to $100 \mathrm{kHz}$ (a home-made portable ultrasonic generator has been developed to feed ultrasonic transducers). A typical scanning mesh was $100 \times 100$ points. In fact, the idea of experiments has been to determine resonance frequencies in each defect situation that would allow significant enhancement of energy generation in the defects thus facilitating the use of ultrasonic IR thermography. Resonance frequencies with a good accuracy ( \pm 10 $\mathrm{Hz}$ ) can be determined by applying a method of laser vibrometry (see the experimental setup in Fig 1c), and further stimulation of resonant vibrations in defects allows their detection under fairly low ultrasonic load.

\section{SAMPLES AND RESULTS}

Four reference samples made of GEC were produced to implement some typical defects which have been anticipated to appear during the manufacturing of TVS-2DTS airplane components (see preliminary results in [4]). One real component (rib) also made of GEC was suspected to have a vast inter-layer crack before the inspection.

The first reference sample having the size of $300 \times 300 \times 2.3 \mathrm{~mm}$ contained four $100 \times 100 \mathrm{~mm}$ inserts made of polyethylene and paper and placed at two depths (Figure 2a). Such foreign inserts may appear as a result of poor workmanship at a manual stage of production where polyethylene and paper are used as packing materials for GEC prepregs. The sample has been tested in a two-side procedure since it has been allowed by production conditions. By analogy with ultrasonic NDT, it can be assumed that a two-sided procedure is typically more sensitive to defects which may appear at any depth in a material. Note that a one-sided procedure of active thermal NDT is very efficient for detecting shallow defects. In our case, by processing a recorded image sequence $\boldsymbol{T}(\boldsymbol{x}, \boldsymbol{y}, \boldsymbol{\tau})$, where $\boldsymbol{x}, \boldsymbol{y}$ are the coordinates, and $\boldsymbol{\tau}$ is the time, it has been possible to produce a single image of material thermal diffusivity $\boldsymbol{a}$. The Parker flash method has been used in its classical form (omitted here) to calculate pixel-based $\boldsymbol{a}$ values, even if the duration of heating with halogen lamps has been $3 \mathrm{~s}$ rather than really pulsed. The corresponding diffusivity map is shown in Figure $2 b$. Two paper inserts can be clearly detected while the indications of polyethylene are vague (Figure $2 \mathrm{~b}$ ). The same defects have been well seen in the thermal tomogram obtained in a one-sided test procedure (Figure 2c). Phase-array ultrasonics tomography and $\mathrm{X}$ ray imaging have been chosen as alternative techniques to compare to thermal NDT results. The quality of $X$ ray images has not been high, perhaps, because of the nature and thickness of inserts (Figure 2d). Ultrasonic tomograms shown in Figure 2e,f provide contrast images of paper inserts and weak indications of polyethylene. In fact, the obtained test results witness that polyethylene inserts may be damaged (melted, etc.) in the process of sample manufacturing. This aspect deserves additional treatment.

The second sample represented in fact a fragment of a TVS-2DTS wing (see Figure 1a and Figure 3a). The thickness of the test panel which was strengthened by stringers and contained some paper and polyethylene inserts varied from 2.26 to $11.3 \mathrm{~mm}$. A complicated geometry of the wing created certain problems while optically heating its surface and required the use of some cutting-edge processing algorithms implemented in the ThermoFit Pro software from Tomsk Polytechnic University. For example, the correlogram in Figure $3 b$ shows a paper insert in the bottom section of the panel. 

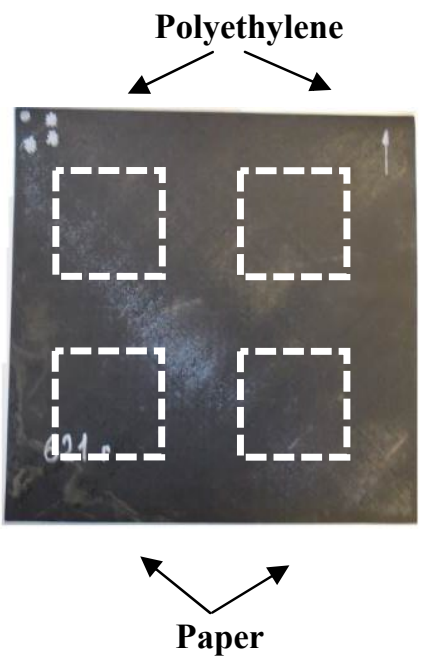

a)

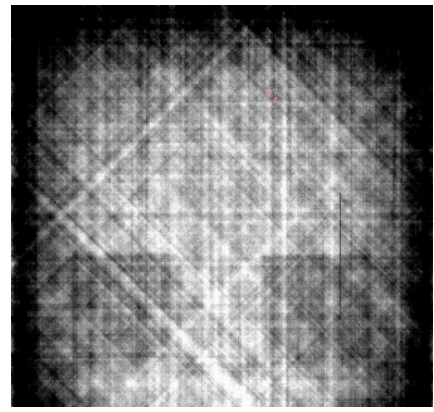

d)

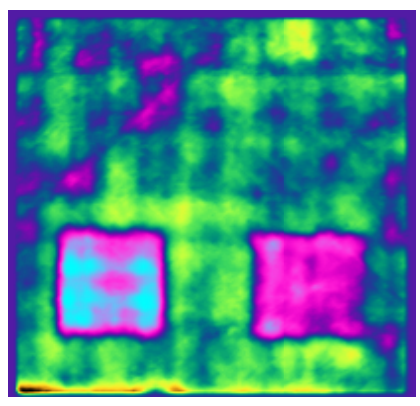

b)

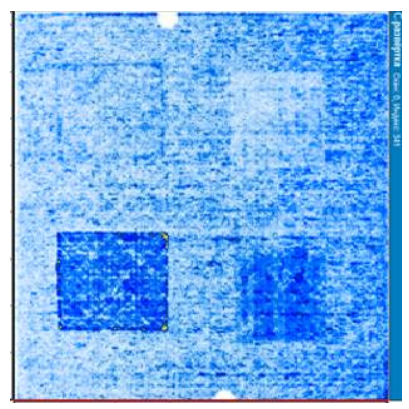

e)

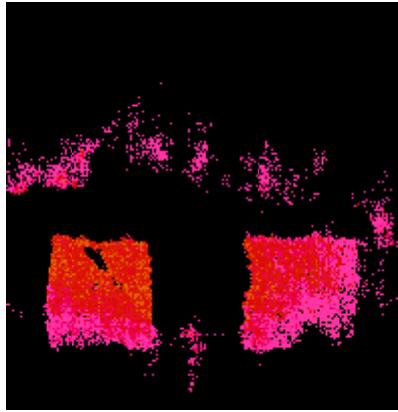

c)

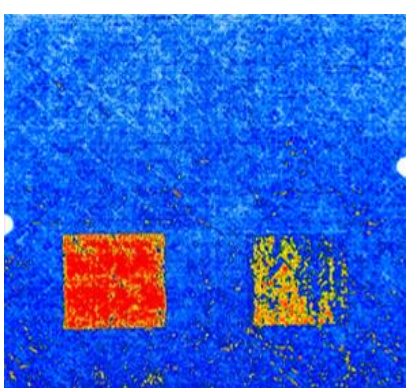

f)

Figure 2. Inspecting GEC reference samples with 4 foreign inserts: $a$ - sample photo , b-diffusivity map (optical stimulation), $\mathrm{c}$ - thermal tomogram, 0.3-0.9 mm layer (one-sided procedure); $\mathrm{d}-\mathrm{X}$ ray image $(100 \mathrm{kev}), \mathrm{e}-$ ultrasonic tomography, 1.9-2.2 mm layer, e - ultrasonic tomography, 0.4-0.6 mm layer.

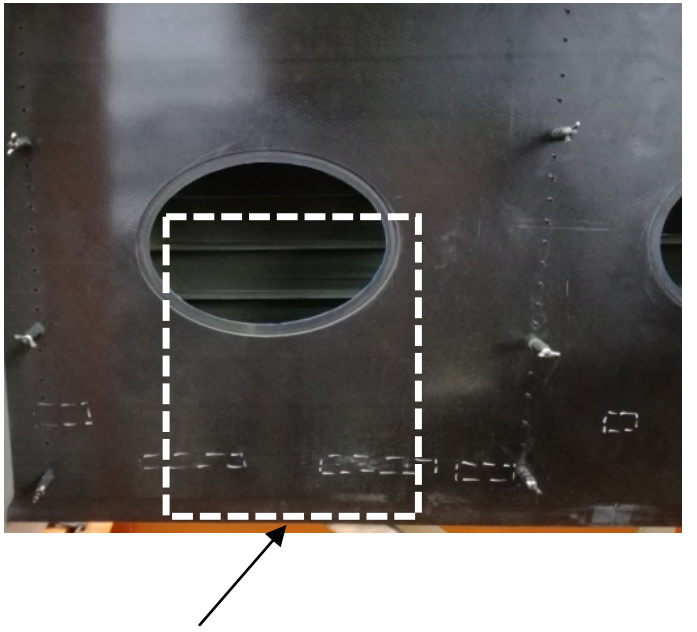

Inspected area

a)

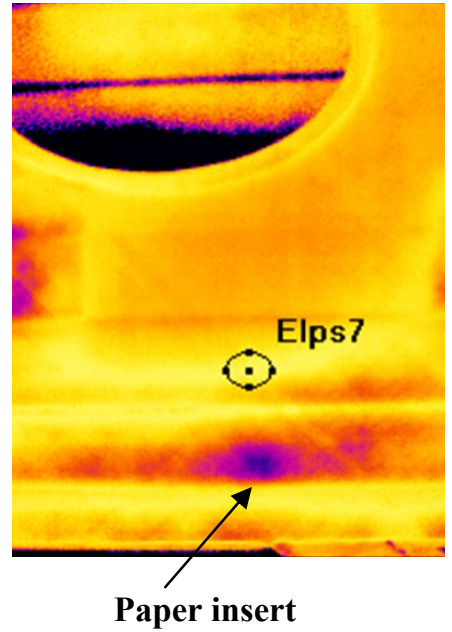

b)

Figure 3. Inspecting GEC wing area-by-area: $a$ - photo of wing fragment, $b$ - correlogram of paper insert. 
Potentials of ultrasonic IR thermography have been demonstrated on two reference samples which contained some Teflon inserts between prepreg layers in the sample central section. As clearly seen in Figure 4, ultrasonic stimulation causes a noticeable friction in the defect sites thus producing local temperature elevations with amplitude about $2^{\circ} \mathrm{C}$ (stimulation with $22 \mathrm{kHz}$ waves for $5 \mathrm{~s}$, electric power $300 \mathrm{~W}$ ).
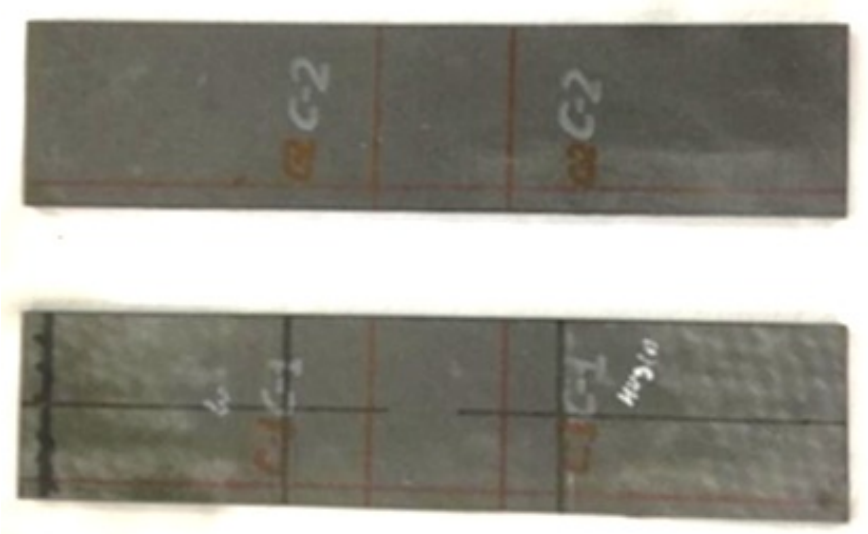

a)
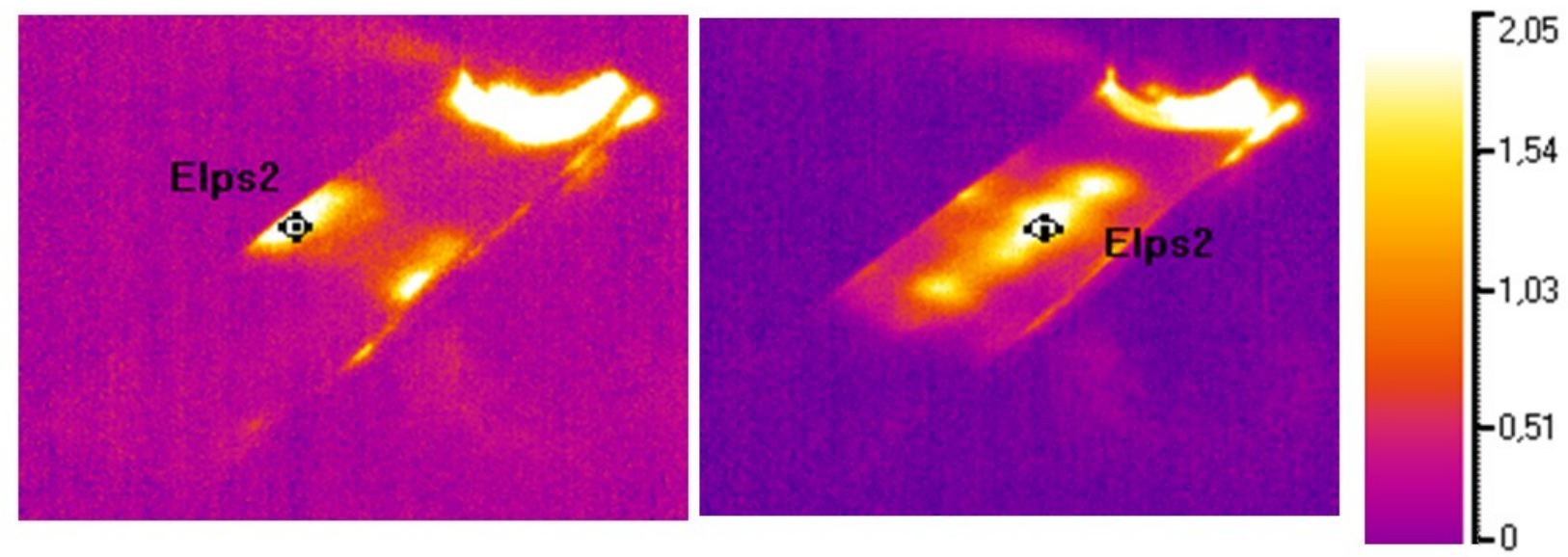

b)

Figure 4. Inspecting GEC reference samples with Teflon inserts by using $5 \mathrm{~s}$ ultrasonic stimulation $(22 \mathrm{kHz}, 300 \mathrm{~W})$ : $\mathrm{a}$ - photo of two samples, $\mathrm{b}$ - IR thermograms.

The fifth test sample - an airplane rib also fully made of GEC (Figure 5a) - was incidentally damaged in a workshop, and some cracks/delaminations were anticipated to develop in it. Both optical and ultrasonic stimulation have been applied to inspect this sample. A two-sided Parker's method was implemented by illuminating and viewing stiffeners under a certain angle in order to visualize contact zones between the stiffeners and the rib. The diffusivity map shown in Figure 5b clearly shows the delaminated stiffener. Even more illustrative have been the results of ultrasonic IR thermography. In order to obtain a 'uniform' picture of the rib, ultrasonic waves were inputted in each particular stiffener to result in the composed IR thermogram shown in Figure 5c. It is evident that the delamination that is also seen in the diffusivity map of Figure $5 b$ occupies a large area under the defective rib. An additional result of ultrasonic IR thermography has been a distinct visualization of "defective holes" where drilling possibly caused some in-hole side cracks (Figure 5c). 


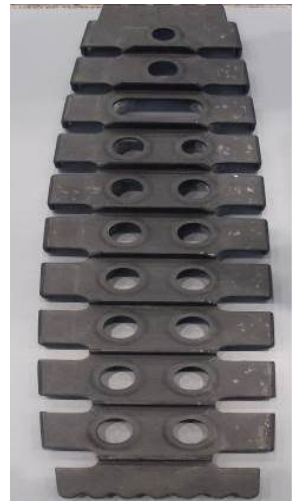

a)

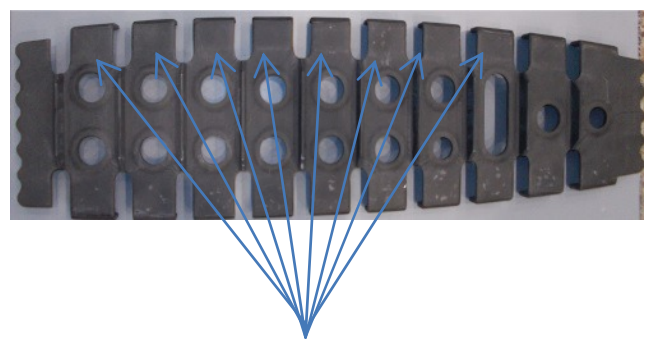

Ultrasonic stimulation points

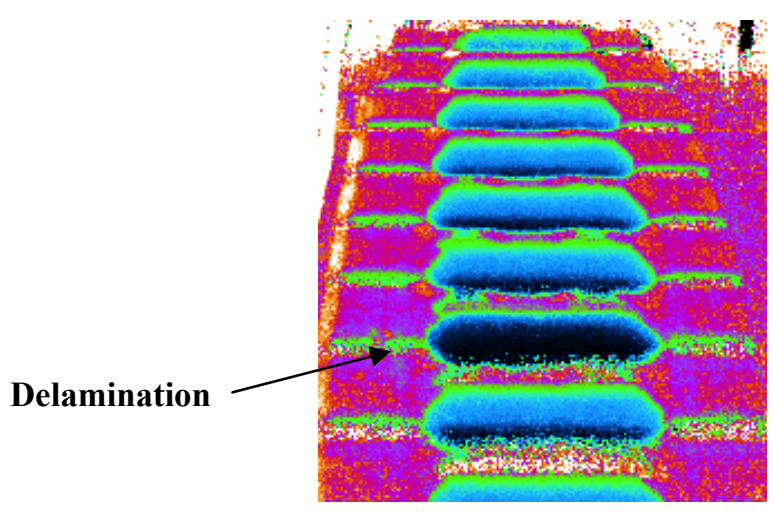

b)

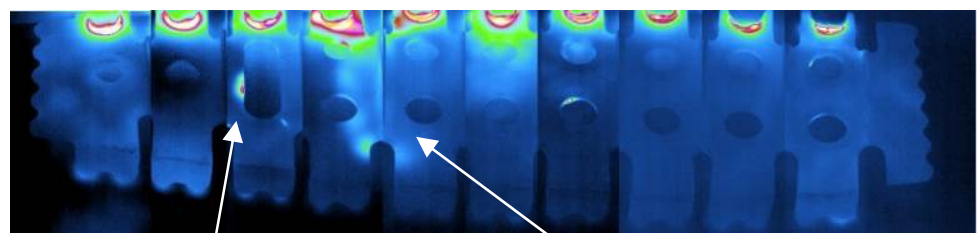

Crack

Delamination

c)

Figure 5. Inspecting GEC rib: $\mathrm{a}$ - sample photo, $\mathrm{b}$ - diffusivity map (two-sided test, optical stimulation), $\mathrm{c}-$ ultrasonic IR thermography (left: points of stimulation, right: composed IR thermogram).

A very illustrative have seemed to be images of surface vibration velocities obtained by applying the technique of laser vibrometry. For example, in the case of the GEC rib, the analysis of sample frequency spectra allowed to determine a resonance frequency of the sample equal to $3.97 \mathrm{kHz}$. At this frequency, the distribution of vibration velocities shown in Figure 6 distinctly reveals the extension of the delamination.

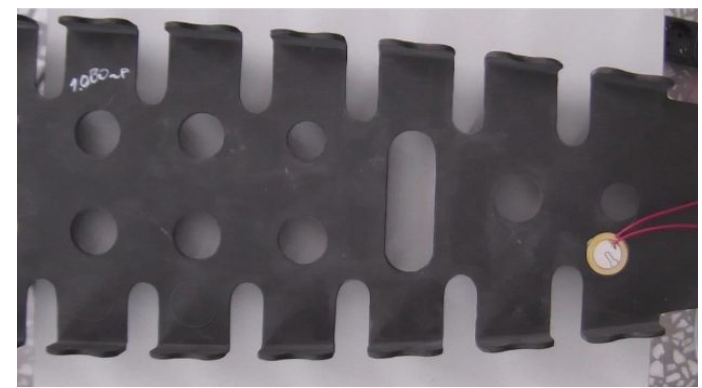

a)

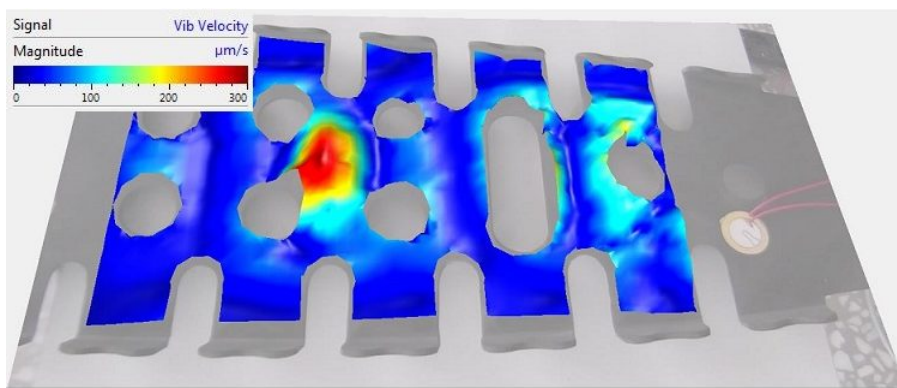

b)

Figure 6. Applying LDR inspection technique in the inspection of airplane GEC rib: a - photo of sample with piezoelectric transducer on the right, $\mathrm{b}$ - distribution of surface vibration velocities at resonance frequency $3.97 \mathrm{kHz}$. 


\section{CONCLUSION}

It has been demonstrated that active thermal NDT may serve as a valuable tool in the evaluation of aviation GEC components in workshop conditions where the manufacturing technology allows both one- and two-sided access to inspected parts. A "mild" optical stimulation seems to be a flexible heating technique, in particular, if some measures against reflected radiation are to be undertaken. However, optical heating might be ineffective on parts with a complicated geometry. In this case, a technique of ultrasonic IR thermography may be useful but its application in a workshop requires the solving of some practical issues related to the necessity of a good and non-harmful contact between the transducer and material. The laser vibrometry technique is very promising for a structural analysis of composites but it is expensive and difficult to apply at a manufacturing stage.

This work was supported by the Russian Scientific Foundation grant \#17-19-01047 (thermal tomography experiments) and in part by State Order of the Russian Ministry of Higher Education for 2017-2019, NIR \#9.5966.2017/BY (experimental setup development).

\section{REFERENCES}

[1] Shah D.U., "Developing plant fibre composites for structural applications by optimising composite parameters: a critical review", J. Mater. Sci., Vol. 48, p. 6083-107 (2013).

[2] Staszewski W.J., Boller C. and Tomlinson G.R., Editors, "Health monitoring of aerospace structures", Chichester, UK, John Wiley \& Sons, Ltd. (2003).

[3] Solodov I., J. Bai J., Bekgulyan S. and Busse G., "A local defect resonance to enhance acoustic wave-defect interaction in ultrasonic nondestructive testing", Appl. Phys. Lett., Volume 99, Issue 21, article number 211911 (2011).

[4] Chulkov A.O., Vavilov V.P., Serieznov A.N. and Bragin A.A., "Developing guidelines for one- and two sided active thermal NDT of graphite/epoxy components of a TVS-2DTS airplane", Proc. 10 ${ }^{\text {th }}$ All-Russia TESTMAT Conf. "Trends and perspectives of nondestructive testing in the aerospace industry", Moscow, Russia, p. 350-360 (9 Febr. 2018) (in Russian). 